\title{
Propuesta metodológica para valorar la calidad escénica de los paisajes del agua y su potencial como recurso turístico
}

\author{
Methodological proposal to evaluate the scenic quality of waterscapes \\ and their potential as a touristic attraction
}

\author{
Josep Pueyo-Ros ${ }^{1}$ (iD \\ Rosa M. Fraguell ${ }^{2}$ (1) \\ Anna Ribas ${ }^{3}$ (ID
}

\section{Resumen}

Los paisajes del agua en destinos turísticos costeros pueden ayudar a reducir la saturación y estacionalidad que sufren este tipo de destinos basados en el recurso playa. A la vez, el turismo puede ofrecer argumentos para la conservación de estos paisajes, a menudo olvidados, creando una relación simbiótica entre turismo y conservación. Para ello se hace necesaria una valoración y tipificación de las cualidades de estos espacios para convertirse en recursos turísticos. En este artículo presentamos una metodología para valorar simultáneamente la calidad escénica de los paisajes y su potencial de atracción turística. La calidad escénica se enfoca con una perspectiva multisensorial, considerando no sólo el paisaje visual, sino también el sonoro y el olfativo. Para evaluar el potencial turístico se valoran aspectos como la relevancia, accesibilidad, calidad de los equipamientos y viabilidad a largo plazo. La metodología es aplicada y testada en tres paisajes del agua del destino turístico Costa Brava (Girona, España), demostrando suficiente fiabilidad para ser extrapolada a otros paisajes del agua.

Palabras clave: Paisajes del agua; turismo de naturaleza; calidad escénica; recurso turístico; paisaje sonoro; paisaje olfativo.

\begin{abstract}
Waterscapes in coastal tourist destinations can reduce overcrowding and seasonality, which effect tourist destinations that focus on beaches as a tourism resource. Furthermore, tourism can justify the conservation of these landscapes, which is often forgotten by urban planners, thus forming a symbiotic relationship between tourism and conservation. To this end, it is necessary to evaluate and classify the attributes of waterscapes so that they can become a tourism resource. In this paper, a method to simultaneously evaluate the scenic quality and the potential as a tourism attraction is presented. Scenic quality focuses on a multisensory perspective, one that not only considers visualscape but also soundscape and smellscape. To evaluate its tourist potential, the relevance, accessibility, equipment and long-term viability were all taken into consideration. The method is applied and tested in three waterscapes of the Costa
\end{abstract}

\footnotetext{
1 Departamento de Geografía. Universidad de Girona, España. josep.pueyo@udg.edu

2 Departamento de Geografía. Universidad de Girona, España. rosa.fraguell@udg.edu

3 Departamento de Geografía. Universidad de Girona, España. anna.ribas@udg.edu
} 
Brava (Girona, Spain). It proved to be reliable enough from an all-round point of view to be extrapolated in other waterscapes.

Keywords: Waterscapes; nature-based tourism; scenic quality; tourism resource; soundscapes; smellscapes.

\section{Introducción}

La severa transformación que ha afectado el litoral del Mediterráneo occidental en las últimas décadas ha provocado una saturación del propio litoral como recurso turístico. Un síntoma fue, por ejemplo, que en la década de 1990 diferentes autores concluyeran que los destinos especializados en turismo de sol y playa en esta área geográfica estaban entrando en lo que Richard W. Butler (1980, 2006, 2011) definió como "fase de estancamiento del ciclo de vida para destinos turísticos maduros" (Sardà, Mora, Ariza, Avila, \& Jimenez, 2009). A partir de aquí, las estrategias que han desarrollado los destinos turísticos con el fin de asegurar su supervivencia han sido diversas. Una de ellas ha sido explorar alternativas complementarias, buscando complicidades con recursos del interior como espacios naturales, elementos patrimoniales, paisajes rurales o simplemente lugares al margen de la transformación urbanística que han vivido las áreas costeras (Cebrián \& García, 2010). Estos recursos del interior permiten a los destinos litorales ofrecer nuevas atracciones a los visitantes a la vez que reducen la saturación del recurso playa y la estacionalidad crónica fruto de la dependencia climática.

De entre los grandes protagonistas de este proceso de búsqueda de recursos complementarios, en este estudio centramos la atención en los paisajes del agua de las llanuras aluviales litorales, que se articulan alrededor de ríos, lagunas o marismas. Los espacios con presencia de agua tienen un interés especial para los turistas, que se han convertido en cazadores de paisajes, buscando la fotografía perfecta (Urry, 2002). En esta fotografía, la presencia del agua es un elemento imprescindible, porque los paisajes con agua son los preferidos por los turistas (Burmil, Daniel, \& Hetherington, 1999; Herzog, 1985). En un artículo sobre los valores de la cuenca del río Baker (Argentina), los autores concluyen que "los paisajes del agua son paisajes esenciales de un territorio, son la expresión sintética de las características geográficas, escenarios de vida y portadores de identidad" (Muñoz, Pérez, Sanhueza, Rovira, \& Urrutia, 2006). En otro artículo sobre los paisajes de la Huerta de Murcia, los autores citan a Martínez de Pisón para enfatizar el valor simbólico del susodicho elemento: "el agua es clave en la relación con el territorio, cargada de simbolismo, un eje que ordena el mundo" (Mata \& Fernández, 2010). Así pues, queda patente la importancia cultural y simbólica del agua en el paisaje. Como consecuencia, la mayoría de estudios dedicados a valorar la calidad escénica de los paisajes concluyen que la presencia de agua es un elemento altamente significativo en su valoración (Arriaza, Cañas-Ortega, Cañas-Madueño, \& Ruiz-Aviles, 2004; Dramstad, Tveit, Fjellstad, $\&$ Fry, 2006; Real, Arce, \& Manuel Sabucedo, 2000).

Los paisajes del agua han sido ampliamente estudiados desde la perspectiva ambiental (Van den Broeck, Waterkeyn, Rhazi, Grillas, \& Brendonck, 2015) y desde la perspectiva de los servicios ecosistémicos (Söderqvist, Mitsch, \& Turner, 2000). En este último caso, se ha observado que las funciones de recreación y turismo son muy importantes y están ligadas a su preservación, tal como se reflejó en la convención RAMSAR del año 2012, donde se reconocía el papel activo del turismo en la conservación de les zonas húmedas (Dhakal, 2014). Por esta razón, es necesario aunar en esta línea de investigación, valorando las cualidades que tienen los paisajes del agua para atraer turismo de litoral. Es necesario complementar la valorización ambiental con la valorización estética y recreativa. Medir el potencial de estos espacios para convertirse en atracciones turísticas capaces de complementar el recurso playa, todo ello con la finalidad de que la actividad turística colabore con la conservación y mejora de los valores ambientales de los paisajes del agua del litoral.

Sin embargo, algunos autores afirman que la turistificación de un espacio natural puede conllevar su mercantilización (Büscher \& Fletcher, 2016) y, por consiguiente, ser contraproducente a la propia conservación del espacio (Fletcher, 2009). Por este motivo, cuando se pone un espacio en valor es importante tener presente que la valorización turística no tiene por qué coincidir con la importancia ecológica del espacio (Gobster, Nassauer, Daniel, \& Fry, 2007). Por lo que los estudios de valoración deben tener presente que funciones o atributos son los que se están valorando y ser conscientes que este no es nunca el valor total del espacio natural valorado (Chan, Satterfield, \& Goldstein, 2012). 
Centrándonos en la valoración estética, los estudios que valoran la calidad escénica de un paisaje suelen limitarse a la componente visual (Daniel, 2001), pero los paisajes también son escuchados (Schafer, 1977) y olidos (Henshaw, 2013). Los paisajes sonoros últimamente están recabando la atención de investigadores del paisaje (Farina, 2014; Iglesias Merchan, Diaz-Balteiro, \& Soliño, 2014; Kim \& Shelby, 2011; Łowicki \& Piotrowska, 2015), pero los paisajes olfativos continúan siendo sistemáticamente olvidados en los estudios de percepción, a pesar de la importancia que tienen en las experiencias vividas por los turistas (Dann \& Jacobsen, 2003) o para la población local (Borer, 2013). Solamente cuando un paisaje es mirado, escuchado y olido podemos disfrutarlo con plenitud; si uno de los tres componentes no es coherente con el conjunto, la valoración de este paisaje puede variar de forma significativa (Hetherington, Daniel, Brownt, Forest, \& Station, 1993).

Independientemente de la dimensión del paisaje que se evalúe, los estudios se pueden clasificar en dos grandes grupos: los basados en criterio experto y los basados en la percepción de los individuos (Daniel \& Boster, 1976). Los primeros basan la valoración de los paisajes en el criterio de un panel de expertos que extraen los valores objetivables del paisaje y los valoran según lo que se considere un paisaje de calidad. Los segundos obtienen la valoración a partir de la realización de encuestas o la organización de grupos de discusión donde se pide a la población encuestada que valore determinados paisajes, ya sea mediante representaciones gráficas encuestando a un grupo reducido de personas o haciendo la encuesta a los visitantes del lugar delante el propio paisaje (ver Mercado-Alonso, Fernández-Tabales, \& Muñoz-Yules (2017) y Nogué, Puigbert, Sala, \& Bretcha (2010) para más información). Los estudios basados en criterio experto han sido muy criticados por el sesgo que pueden provocar, ya que las valoraciones son siempre subjetivas, aunque sean de expertos. Los estudios basados en la percepción generan resultados más fiables pero necesitan más recursos para llevarse a cabo (Daniel, 2001). Por este motivo, diferentes académicos se han dedicado a buscar correspondencias entre los resultados de los dos tipos de estudio, buscando relaciones, por ejemplo, entre indicadores de ecología del paisaje con la valoración estética de los visitantes (de la Fuente de Val, Atauri, \& de Lucio, 2006). Desafortunadamente, estos estudios centran la atención solamente en la dimensión visual de los paisajes. Otro problema que presentan es que no esclarecen como solucionar la divergencia entre los resultados obtenidos entre uno y otro tipo de estudio (Daniel, 2001). Aun así, una revisión de los estudios realizados en esta línea puede ayudar a definir los criterios que se repiten como significativos y que, por lo tanto, se pueden considerar criterios estables o universales.

En lo que se refiere a los paisajes sonoros, los resultados de estudios previos coinciden en valorar como positivas las biofonías (sonidos originados por seres vivos no humanos) y las geofonías (sonidos originados por elementos inertes como el viento, el agua, etc.) y como negativos las antropofonías (sonidos originados por la actividad humana). Así, por ejemplo, existen ejemplos de cómo las antropofonías nocturnas afectan el precio de las viviendas (Łowicki \& Piotrowska, 2015). También como la presencia de biofonías y geofonías reducen la percepción de saturación en espacios naturales, mientas que las antropofonías la incrementan (Kim \& Shelby, 2011). Otro ejemplo se puede encontrar para el caso de estudio del parque natural de Peñalara, en Madrid, donde se concluyó que los turistas estarían dispuestos a pagar un $1 €$ de promedio por visita con el fin de colaborar en la financiación de un programa de reducción de ruidos (Iglesias Merchan et al., 2014). En relación al turismo ornitológico, muy significativo en determinados paisajes del agua, la relación no es tan directa como en los casos anteriores. Mientras los ornitólogos aficionados valoran igual biofonías y geofonías, estas últimas son menos valoradas a medida que aumenta la experiencia de los turistas. La valoración negativa en relación a las antropofonías se mantiene constante independientemente de la experiencia del ornitólogo. Prior (2016) detalla muchos más casos de estudio en su revisión sobre el análisis de los paisajes sonoros, donde se llega siempre a la conclusión que son preferibles los sonidos naturales por encima de los antrópicos en los paisajes "naturales".

Por el contrario, hasta donde llega nuestro conocimiento, no hay estudios de percepción basados en encuestas para los paisajes olfativos. Se puede encontrar, de todas formas, una revisión de la literatura sobre viajes con el fin de evaluar el papel que desempeñan los paisajes olfativos en los destinos turísticos. El estudio muestra que los olores de los paisajes rurales son mejor valorados que los de los paisajes urbanos, aunque esta tendencia disminuye cuando la literatura hace referencia a paisajes urbanos posmodernos (Dann \& Jacobsen, 2003). Otros estudios sobre paisajes olfativos se limitan a analizar los paisajes olfativos urbanos, ya sea desde una perspectiva sociológica (Borer, 2013; Sliwa \& Riach, 2011) o de planifica- 
ción urbana (Henshaw, 2013). Así pues, los estudios de percepción en paisajes no urbanos son todavía un campo de conocimiento por explorar.

Para que un paisaje se convierta en un recurso turístico no es suficiente con que cuente con una gran calidad escénica, sino que también es necesario que sea visitado por turistas (Lew, 1987). Y para que haya turistas es necesario que el espacio disponga de las cualidades necesarias para atraerlos, como serían la accesibilidad (Kušen, 2010), la promoción (Ferrario, 1979) o la disponibilidad de equipamientos (Navrátil \& Pícha, 2013). Así pues, para la valorización turística de un paisaje se tiene que considerar tanto su calidad escénica como su capacidad de atracción como recurso turístico.

Siguiendo esta línea, este trabajo tiene por objetivo presentar una propuesta metodológica para la valorización turística de los paisajes del agua. La metodología es aplicada y testada en tres casos de estudio de la Costa Brava (Girona). El estudio centra la atención en la calidad escénica de los paisajes desde un enfoque multisensorial (visual, sonoro y olfativo) y también desde su potencial como recurso turístico. Considerar estos dos aspectos de un paisaje permite obtener una tipificación de los paisajes del agua útil para la gestión de destinos turísticos.

\subsection{Casos de estudio}

Los tres paisajes del agua elegidos para aplicar y testar la metodología propuesta se localizan en el destino turístico Costa Brava (Girona, España). La Costa Brava es un destino centrado en el turismo de sol y playa, donde la disponibilidad de playas constituye su recurso esencial (Sardà et al., 2009). Por lo tanto, la Costa Brava constituye un buen emplazamiento donde estudiar como los paisajes del agua litorales que se articulan alrededor de ríos, lagunas o marismas pueden ayudar a reducir tanto la estacionalidad del turismo como la saturación del recurso playa.

El primero de los paisajes del agua elegidos es "les Basses del Ter Vell". Se trata de un espacio protegido ubicado en la antigua desembocadura del río Ter. El paisaje está formado esencialmente por dos lagunas de agua dulce de media hectárea cada una rodeadas de una extensión continua de carrizo (Phragmites australis) que ocupa aproximadamente unas 18 ha. Se trata de una reserva natural parcial (categoría IV según la clasificación de la UICN) integrada, como las otras dos, en el parque natural del "Montgrí, Illes Medes i Baix Ter" (categoría V de la UICN). Sólo puede visitarse desde caminos circundantes o desde una caseta de observación situada en frente de la laguna principal. El principal interés para su elección se debe a que se trata de un espacio protegido colindante con el núcleo turístico de l'Estartit.

Figura 1. Localización de los paisajes del agua seleccionados para el estudio
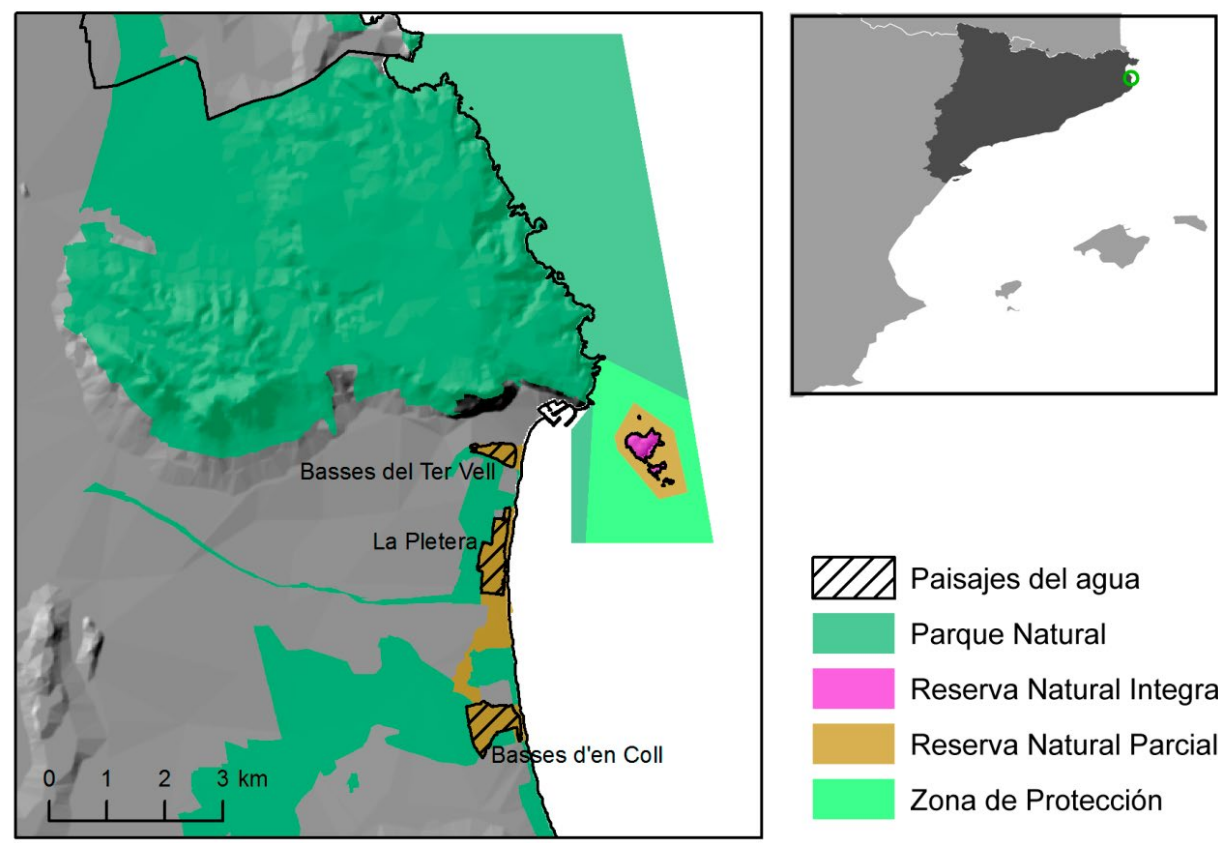

Paisajes del agua

Parque Natural

Reserva Natural Integral

Reserva Natural Parcial

Zona de Protección

Fuente: Institut Cartogràfic i Geològic de Catalunya [ICGC] (2016). Elaboración propia. 
El segundo paisaje elegido es "la Pletera", un paisaje antaño formado por una extensión de salicornia y también protegido bajo la figura de reserva natural parcial. El espacio fue víctima del intenso proceso de urbanización del litoral, pero el proyecto quedó parado en la década de 1990 después de la quiebra de la empresa promotora. Sólo se llegaron a construir las calles, un paseo peatonal y una manzana de casas de segunda residencia, lo que convirtió el espacio restante en una amalgama de parcelas de salicornia (las más cercanas a la playa) combinadas con parcelas de matorrales típicos de tierra baja que ocupa en total unas 45 ha. La inclusión de este paisaje del agua degradado ha de permitir testar como la metodología presentada funciona con este tipo de paisajes desde la perspectiva de su valorización turística.

Finalmente, el tercer paisaje objeto de estudio es "les Basses d'en Coll", una laguna de 3,5 ha rodeada de unas 41 ha de arrozales y carrizo. También goza de la figura de reserva natural parcial y dispone de varios caminos que atraviesan los arrozales y que permiten acceder a la laguna, así como una caseta de observación. Es un espacio eminentemente rural que contrasta con los dos anteriores y donde el agua está mucho más presente, no sólo por la gran laguna sino también por la red de regadío que conduce el agua a los arrozales.

Figura 2. Vistas aéreas de los tres paisajes del agua objetos de estudio. De izquierda a derecha: "La Pletera", "Les Basses del Ter Vell" y "les Basses d'en Coll".

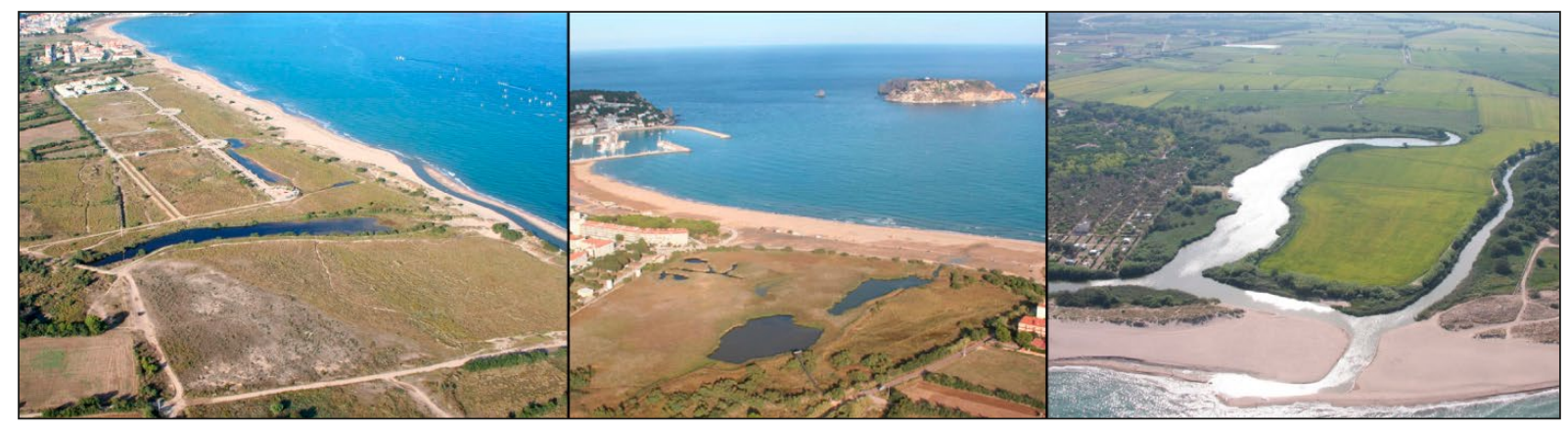

Fuente: http://ramonfortia.cat (2016).

\section{Metodología}

Como ya se ha comentado, para valorar la calidad escénica de un paisaje no es suficiente con evaluar la dimensión visual. Es necesario considerar también las dimensiones sonora y olfativa. Puesto que son tres aspectos muy diferentes entre sí, se diseñó un método particular para cada dimensión.

\subsection{Evaluando el paisaje visual}

La revisión bibliográfica de los estudios que se han dedicado a buscar correspondencias entre atributos objetivables del paisaje y resultados de los estudios de percepción permitió extraer los atributos más significativos para evaluar la calidad escénica de un paisaje. En total se revisaron 15 artículos (todos los que se encontraron en la base de datos "Scopus" con la temática mencionada) de los cuales se seleccionaron los atributos que se mostraban significativos en tres o más artículos y que no aparecían como no significativos en ningún otro, a fin de excluir los resultados divergentes entre estudios. Cinco atributos cumplían estas dos condiciones: superficie de la cuenca visual, presencia de agua, diversidad, grado de naturalidad y la percepción de misterio, una de las variables del modelo cognitivo de Rachel \& Stephen Kaplan (1989) (tabla 1).

Para calcular estos atributos para cada paisaje se partió de la metodología utilizada por Schirpke, Tasser, \& Tappeiner (2013) para valorar la belleza escénica en paisajes de montaña. En primer lugar se utilizó un Sistema de Información Geográfica (SIG) para situar puntos de observación aleatorios en los caminos que los visitantes utilizan para recorrer el espacio. Puesto que nuestro objeto de estudio son paisajes de escala local y de relieve plano, optamos por localizar un punto cada 100 metros más otros puntos en lugares significativos (puntos elevados y puntos de información). Para cada punto se calcularon las cuencas visuales considerando el relieve y las alturas de las cubiertas, ya que en paisajes planos las cubiertas son el principal obstáculo en la línea de visión. Se situó el observador a una altura de 1,60 m (Schirpke et al., 2013). 
La superficie de la cuenca visual $(s c v)$ es el promedio de las superficies en quilómetros cuadrados de la cuenca visual desde cada punto de observación. Para cuantificar la presencia de agua (agu) se calculó el porcentaje de puntos de observación desde los cuales se podía ver una lámina de agua. Para la diversidad, algunos estudios utilizan el índice de Simpson (de la Fuente de Val et al., 2006; Schirpke et al., 2013) y otros el índice de Shannon (Dramstad et al., 2006). En nuestro estudio preferimos utilizar el índice de diversidad de Simpson (div), ya que genera resultados entre 0 (mínima diversidad) y 1 (máxima diversidad) facilitando la comparación entre paisajes:

$$
d i v=1-\sum_{i=1}^{n} p_{i}^{2}
$$

Donde $p_{i}$ es la proporción de la superficie que ocupa la clase $i$ respecto la superficie total. Para complementar la medida de diversidad también consideramos la diversidad de colores visibles des de los puntos de observación (Arriaza et al., 2004; de la Fuente de Val et al., 2006), partiendo de los colores básicos (amarillo, verde, azul, rojo, blanco y negro) se agruparon las cubiertas del suelo en función del color que presentan los meses de máxima afluencia turística (i.e. los meses de verano). Con estas nuevas categorías se calculó un nuevo índice de Simpson ( $\mathrm{col}$ ). Los índices de diversidad son susceptibles a la cantidad de clases, por consiguiente, para poder comparar diferentes paisajes es necesario utilizar la misma clasificación de cubiertas del suelo. Para nuestro estudio utilizamos el nivel 2 del mapa de cubiertas del suelo de Catalunya a escala 1:2500 realizado por el Centre de Recerca Ecologica i Aplicacions Forestals [CREAF] (Ibàñez \& Burriel, 2010). Para calcular el grado de naturalidad (nat) muchos estudios utilizan variables categóricas que dependen de la subjetividad del experto (Arriaza et al., 2004; Real et al., 2000). Para evitar el posible sesgo, se decidió utilizar la proporción de cubiertas que no fueran artificiales que se encontraban dentro de la cuenca visual de los puntos de observación. Como cubiertas artificiales se incluyeron las áreas urbanas, las construcciones aisladas y las carreteras asfaltadas. Los elementos del patrimonio cultural (los únicos que se encontraron en el área de estudio fueron edificios religiosos) y los espacios antrópicos naturalizados (como vías verdes viarias o parques urbanos) no se consideraron dentro de la categoría de artificiales porque no generan impactos visuales negativos (Arriaza et al., 2004). La última variable a considerar fue la percepción de misterio (mis), que como se mencionó, se desprende del modelo cognitivo conocido como modelo de Kaplan y Kaplan. Los autores que han buscado relaciones entre lo que se puede considerar percepción del misterio y atributos objetivos del paisaje la relacionaron con el relieve y con la no presencia humana. Puesto que la presencia humana ya se contempló en la variable anterior, se optó por relacionar la percepción de misterio con el relieve, utilizando la desviación estándar de las elevaciones dentro de la cuenca visual, ya que los elementos ocultos por las diferencias de elevación aumentan la percepción de misterio de los observadores (Germino, Reiners, Blasko, McLeod, \& Bastian, 2001; Gimblett, Itami, \& Fitzgibbon, 1985). Debido a que el estudio está localizado en paisajes llanos, también se consideraron las alturas de las cubiertas naturales del suelo (no se consideraron las antrópicas al ser opuestas a la percepción de misterio).

La nitidez del agua (nit) es un aspecto a tener en cuenta cuando se evalúan paisajes del agua. Los autores que analizaron esta variable concluyeron que cuanto mayor es la nitidez mayor es la calidad escénica de un paisaje del agua (Keeler et al., 2015; Poor, Boyle, Taylor, \& Bouchard, 2001; Wilson \& Carpenter, 1999). Sin embargo, estos estudios están realizados en lagos, donde la nitidez del agua se relaciona con la calidad ecológica. En los paisajes del agua de las llanuras litorales esta relación no tiene por qué ser cierta. Podemos encontrar un ejemplo en el pez fartet (Aphanius Iberus), una especie endémica de la Península Ibérica que habita en aguas de transición propias de las llanuras litorales. El fartet necesita que el agua esté poblada de algas verdes (Alcaraz, Pou-Rovira, \& García-Berthou, 2007) que le dan una apariencia turbia, contraponiéndose la calidad ecológica con la calidad escénica vinculada a la nitidez del agua. Por este motivo se consideró interesante estudiar la percepción que tienen los visitantes sobre la nitidez del agua y analizar si el buen estado ecológico del agua se traduce también en una percepción positiva o si, por el contrario, los turistas valoran favorablemente que el agua sea nítida a pesar de las necesidades ecológicas del espacio. Con el fin de investigar esta cuestión, se preguntó a los visitantes del espacio como percibían la calidad del agua en una escala del 1 (muy mala) al 5 (muy buena). También se les preguntó si su valoración del paisaje mejoraría si el agua fuera más nítida. Cuando la respuesta era afirmativa, se les explicaba que la nitidez podía no ser compatible con la calidad ecológica y se les hacía de nuevo la pregunta. Si la nitidez del agua fuera un factor influyente en la percepción de los visitantes, esta se añadiría al resto de variables utilizadas parar valorar la calidad escénica. 


\subsection{Evaluando el paisaje sonoro}

El aspecto más complejo para evaluar un paisaje sonoro son las técnicas de recogida de datos, utilizando a menudo equipos de grabación de alta fidelidad de elevado coste (Iglesias Merchan et al., 2014). Aun así, estos métodos suelen terminar con una identificación manual de los sonidos registrados durante la recogida de datos. Para reducir la complejidad del proceso, se optó para realizar una identificación manual in situ. De esta manera, los propios encuestadores realizaron una serie de escuchas en diferentes puntos de los espacios y en diferentes horas y días. En cada escucha se anotó si dominaban los sonidos de origen natural o antrópicos. Se otorgó un punto a cada escucha donde dominaban los sonidos naturales, cero puntos si dominaban los sonidos antrópicos y medio punto donde no había un grupo de sonidos dominante. Esta puntuación se definió en base a los resultados de trabajos previos donde los sonidos naturales resultaron ser apreciados por los visitantes mientras los antrópicos resultaron ser sonidos no deseados (Hetherington et al., 1993; Iglesias Merchan et al., 2014; Kim \& Shelby, 2011; Łowicki \& Piotrowska, 2015; Miller, 2001).

Tabla 1. Atributos seleccionados para evaluar la calidad escénica del paisaje

\begin{tabular}{|c|c|c|c|c|c|}
\hline Componente & Variable & Código & Indicador & Fuente de información & Referencias \\
\hline \multirow{7}{*}{ Paisaje visual } & $\begin{array}{l}\text { Superfície de la } \\
\text { cuenca visual }\end{array}$ & vs & $\begin{array}{l}\text { Superficie media de } \\
\text { las cuencas visuales }\end{array}$ & $\begin{array}{l}\text { Modelo digital de } \\
\text { elevaciones + Mapa de } \\
\text { cubiertas del suelo. }\end{array}$ & $\begin{array}{l}\text { (Burmil et al., 1999; Germino et al., } \\
\text { 2001; Herzog, 1985; Kaltenborn \& } \\
\text { Bjerke, 2002) }\end{array}$ \\
\hline & Presencia de agua & $a g u$ & $\begin{array}{l}\text { Probabilidad de ver } \\
\text { una lámina de agua } \\
\text { desde un punto de } \\
\text { observación }\end{array}$ & $\begin{array}{l}\text { Mapa de cubiertas del } \\
\text { suelo. }\end{array}$ & $\begin{array}{l}\text { (Arriaza et al., 2004; Brown \& } \\
\text { Daniel, 1991; Dramstad et al., 2006; } \\
\text { Herzog, 1985; Real et al., 2000) }\end{array}$ \\
\hline & $\begin{array}{l}\text { Diversidad de } \\
\text { cubiertas del suelo }\end{array}$ & $\operatorname{div}$ & $\begin{array}{l}\text { Media del índice } \\
\text { de diversidad de } \\
\text { Simpson para las } \\
\text { cubiertas del suelo } \\
\text { dentro de las cuencas } \\
\text { visuales }\end{array}$ & $\begin{array}{l}\text { Mapa de cubiertas del } \\
\text { suelo. }\end{array}$ & $\begin{array}{l}\text { (de la Fuente de Val et al., 2006; } \\
\text { Schirpke et al., 2013) }\end{array}$ \\
\hline & $\begin{array}{l}\text { Diversidad de } \\
\text { colores }\end{array}$ & col & $\begin{array}{l}\text { Media del índice } \\
\text { de diversidad de } \\
\text { Simpson para los } \\
\text { colores dentro de las } \\
\text { cuencas visuales }\end{array}$ & $\begin{array}{l}\text { Mapa de cubiertas del } \\
\text { suelo. }\end{array}$ & $\begin{array}{l}\text { (Arriaza et al., 2004; de la Fuente de } \\
\text { Val et al., 2006) }\end{array}$ \\
\hline & $\begin{array}{l}\text { Grado de } \\
\text { naturalidad }\end{array}$ & nat & $\begin{array}{l}\text { Proporción de } \\
\text { superficie no artificial } \\
\text { dentro de las cuencas } \\
\text { visuales }\end{array}$ & $\begin{array}{l}\text { Mapa de cubiertas del } \\
\text { suelo. }\end{array}$ & $\begin{array}{l}\text { (Arriaza et al., 2004; Real et al., } \\
\text { 2000; Vila et al., 2015) }\end{array}$ \\
\hline & $\begin{array}{l}\text { Percepción de } \\
\text { misterio }\end{array}$ & mis & $\begin{array}{l}\text { Desviación estándar } \\
\text { media de las altitudes } \\
\text { dentro de las cuencas } \\
\text { visuales }\end{array}$ & Modelo digital del terreno & $\begin{array}{l}\text { (Germino et al., 2001; Real et al., } \\
\text { 2000; Stamps, 2004) }\end{array}$ \\
\hline & Nitidez del agua & nit & $\begin{array}{l}\text { Escala de puntuación } \\
\text { del } 1 \text { al } 5\end{array}$ & $\begin{array}{l}\text { Encuestas a los visitantes } \\
\text { del espacio }\end{array}$ & $\begin{array}{l}\text { (Keeler et al., 2015; Poor et al., } \\
\text { 2001; Wilson \& Carpenter, 1999) }\end{array}$ \\
\hline Paisaje sonoro & $\begin{array}{l}\text { Naturalidad } \\
\text { de los sonidos } \\
\text { escuchados }\end{array}$ & $C S$ & $\begin{array}{l}\text { Dominancia } \\
\text { de biofonías y } \\
\text { geofonías respecto } \\
\text { antropofonías }\end{array}$ & Escuchas in situ & $\begin{array}{l}\text { (Hetherington et al., 1993; Iglesias } \\
\text { Merchan et al., 2014; Kim \& Shelby, } \\
\text { 2011; Łowicki \& Piotrowska, 2015; } \\
\text { Miller, 2001) }\end{array}$ \\
\hline Paisaje olfativo & $\begin{array}{l}\text { Percepción del olor } \\
\text { del espacio }\end{array}$ & $\mathrm{CO}$ & $\begin{array}{l}\text { Escala de puntuación } \\
\text { del } 1 \text { al } 5\end{array}$ & $\begin{array}{l}\text { Encuesta a los visitantes } \\
\text { en el espacio }\end{array}$ & \\
\hline
\end{tabular}

Elaboración propia

\subsection{Evaluando el paisaje olfativo}

Dada la ausencia de estudios previos dedicados a valorar la componente olfativa en espacios naturales, fue necesario realizar un estudio de percepción con el fin de conocer que olores son valorados positivamente y cuales negativamente. En las encuestas se pidió a los encuestados que definieran el olor del paisaje en una palabra. Esta pregunta tenía por objetivo centrar la atención del visitante en el olor que percibía. Una vez la atención estaba puesta en el sentido del olfato, se pedía una valoración del 1 (muy desagradable) al 5 (muy agradable) que fue la que se utilizó para evaluar la calidad olfativa de cada espacio. 


\section{4. Índice sintético de calidad visual del paisaje}

Después de explicar a los participantes que los paisajes son percibidos por la vista, el oído y el olfato, se les pidió que valoraran la importancia de cada sentido en una escala del 1 (muy bajo) al 5 (muy alto). Esto permitió conocer la importancia de cada componente del paisaje con el fin de elaborar un indicador sintético de la calidad escénica donde cada componente se ponderó en función de estos resultados.

Para cada componente del paisaje se obtuvo una puntuación. Para la componente visual se estandarizaron las variables y se generó un indicador sintético de la calidad visual en función de las seis variables calculadas (o siete si se incluyera la nitidez del agua) (tabla 1):

$$
C V=Z_{s c v}+Z_{a g u}+Z_{\text {div }}+Z_{\text {col }}+Z_{\text {nat }}+Z_{\text {mis }} / /+Z_{\text {nit }} / /
$$

Para la calidad sonora, se calculó la puntuación media de todas las escuchas realizadas. De la misma forma, para la calidad olfativa se utilizó la puntuación media obtenida en las encuestas para cada espacio.

El índice de calidad escénica se obtuvo a partir de la suma de las puntuaciones estandarizadas de cada componente, ponderadas en función de la importancia de cada sentido, obtenida en las encuestas:

$$
C E=a Z_{C V}+b Z_{C S}+c Z_{C O}
$$

Donde $Z$ es la puntuación estandarizada de cada componente del paisaje (visual, sonora y olfativa) y $a, b$ y $c$ son las ponderaciones para cada componente.

Para poder comprobar la validez del método, se preguntó a los visitantes que valorasen la calidad del paisaje en una escala del 1 (muy baja) al 5 (muy alta). Los resultados de esta valoración se compararon con los resultados obtenidos con la metodología para comprobar la correlación entre la valoración subjetiva de los visitantes y la valoración obtenida con el método.

\subsection{Calculando el potencial como atracción turística}

Una revisión bibliográfica de los aspectos que valoran los turistas cuando visitan elementos de interés turístico localizados en un espacio natural señala cuatro cualidades que debe cumplir un espacio natural para ser atractivo para los turistas: accesibilidad (Lew, 1987), equipamiento (Navrátil \& Pícha, 2013), grado de protección (Ministerio de Cultura, Empresa y Turismo [MCET], 2008) y relevancia del lugar (Ferrario, 1979). La relevancia de un espacio es importante para que los turistas conozcan su existencia cuando planifican el viaje y genere interés en visitarlo. Con este fin, analizar el nivel de presencia del espacio en cuestión en las guías turísticas resulta ser un buen indicador (Ferrario, 1979). Otro elemento a considerar es la presencia de elementos culturales o patrimoniales que complementen los valores ambientales (Prat \& Cànoves, 2012). Una vez los turistas tienen la motivación para visitar un espacio, es necesario que este sea accesible desde los núcleos donde se localizan los servicios turísticos, la accesibilidad será más alta cuanto más corta sea la distancia desde el núcleo turístico. La señalización para llegar al lugar sin dificultades también es relevante. Durante la visita, es recomendable que el espacio disponga de equipamiento que aumente el interés y el placer de la visita. Los equipamientos que se consideraron fueron: los caminos que permitan recorrer el espacio, el mobiliario donde descansar o comer, los paneles explicativos, los miradores y los centros de información que permiten conocer las peculiaridades del espacio. Finalmente, también se hace necesario garantizar la viabilidad a largo plazo del recurso. Al tratarse de paisajes, la mejor forma de garantizar esta viabilidad es con alguna figura de protección que impida transformaciones que puedan degradar su calidad escénica. A partir de todas estas consideraciones, se utilizó un panel de expertos, compuesto por la mayoría de miembros (un total de 9) del grupo de investigación al cual pertenecen los autores, para definir una variable categórica para cada una de estas características mencionadas (tabla 2).

Por consiguiente, el indicador de cada espacio resultó de la puntuación media obtenida en cada una de las categorías de la tabla 2, con un rango entre 1 y 3 . Variables como la importancia cultural o el estado de los equipamientos dependen en cierta medida de percepciones subjetivas. Para reducir la subjetividad, se consultó a varios expertos para que valoraran desde su propia experiencia cada una de estas categorías. 
Tabla 2. Características a considerar para la valoración del potencial de un paisaje como elemento de atracción turística

\begin{tabular}{|c|c|c|}
\hline Característica & Variable & Categorías \\
\hline \multirow{2}{*}{ Relevancia } & Presencia en guías turísticas & $\begin{array}{l}\text { 1. No aparece en las guías } \\
\text { 2. Aparece en guías locales } \\
\text { 3. Aparece en guías locales e internacionales }\end{array}$ \\
\hline & Importancia cultural & $\begin{array}{l}\text { 1. Sin elementos patrimoniales } \\
\text { 2. Presencia de elementos patrimoniales } \\
\text { 3. Espacio de importancia histórica }\end{array}$ \\
\hline \multirow{2}{*}{ Accesibilidad } & Distancia desde el núcleo más próximo & $\begin{array}{l}\text { 1. Más de } 15 \text { minutos en coche } \\
\text { 2. Más de } 20 \text { minutos a pie } \\
\text { 3. Menos de } 20 \text { minutos a pie }\end{array}$ \\
\hline & Señalización & $\begin{array}{l}\text { 1. Inexistente } \\
\text { 2. Precaria } \\
\text { 3. Buena }\end{array}$ \\
\hline \multirow{3}{*}{ Equipamientos } & $\begin{array}{l}\text { Longitud total de los caminos para visitar } \\
\text { el espacio/Superficie del espacio }\end{array}$ & $\begin{array}{l}\text { 1. Inexistentes } \\
\text { 2. Pocos caminos } \\
\text { 3. Muchos caminos }\end{array}$ \\
\hline & Zona de picnic y bancos & $\begin{array}{l}\text { 1. No hay equipamientos } \\
\text { 2. Equipamientos escasos y/o en mal estado } \\
\text { 3. Equipamientos abundantes y en buen estado }\end{array}$ \\
\hline & Puntos de información & $\begin{array}{l}\text { 1. No hay paneles informativos } \\
\text { 2. Paneles escasos y/o precarios } \\
\text { 3. Paneles abundantes y en buen estado o centro de interpretación }\end{array}$ \\
\hline Viabilidad & Grado de protección & $\begin{array}{l}\text { 1. Sin protección } \\
\text { 2. Red Natura } 2000 \\
\text { 3. Espacio Natural de Protección Especial }\end{array}$ \\
\hline
\end{tabular}

Elaboración propia.

\subsection{Diagrama de priorización de los paisajes del agua}

Una vez calculados los índices de calidad escénica y el potencial como atracción turística de cada espacio, el siguiente paso fue tipificarlos en función de ambas características utilizando un gráfico bidimensional (figura 3). El diagrama se dividió en cuadrantes que permitieron obtener cuatro categorías de espacios. En el cuadrante superior derecho se localizan los espacios con una calidad escénica elevada y con mucho potencial como atracciones turísticas. Se pueden considerar espacios maduros, donde posiblemente lo más interesante es continuar con las tareas de promoción para consolidar el espacio como recurso turístico. En el cuadrante inferior derecho, encontramos espacios con una elevada calidad escénica pero sin las características necesarias para convertirse en una atracción turística. Estos espacios requieren una especial consideración porque con la gestión e inversión necesarias podrían convertirse en importantes recursos turísticos de una destinación. En el cuadrante superior izquierdo se localizan espacios con mucho potencial turístico pero sin calidad escénica destacable. Podrían ser espacios donde ubicar servicios recreativos o deportivos en lugar de potenciarlos por sus valores estéticos. Finalmente, en el cuadrante inferior izquierdo encontramos espacios sin vocación turística, que pueden ser destinados a funciones agrarias o de preservación, como zonas de cría de fauna o de repoblación de especies amenazadas.

Figura 3. Diagrama de tipificación de los paisajes del agua

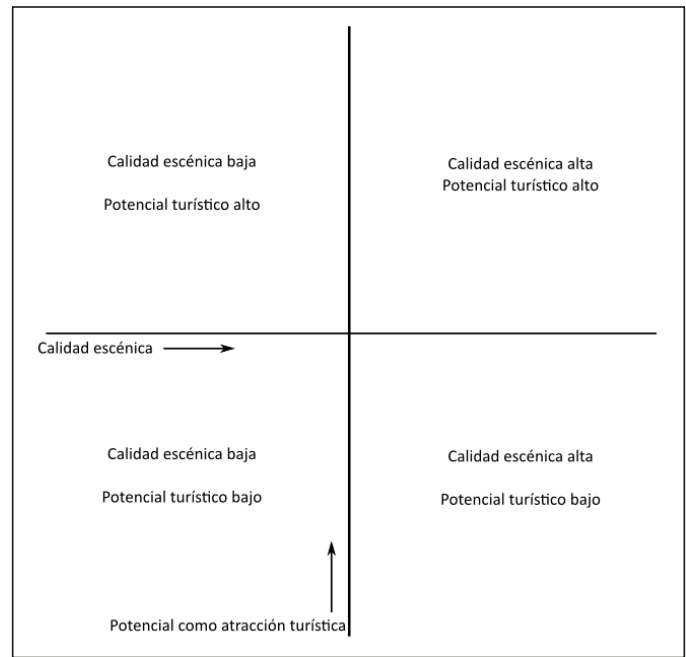

Elaboración propia. 


\section{Resultados}

Durante el proceso de elaboración del estudio se calcularon un total de 64 cuencas visuales repartidas entre los tres espacios estudiados y se realizaron 133 encuestas a visitantes de los espacios, en días soleados, durante el periodo comprendido entre el 15 de junio y el 5 de agosto de 2015, de las 8:00 a las 21:00, de lunes a domingo. Se utilizó el método de intercepción porque es el más apropiado para recolectar información de los visitantes de un espacio natural (Burger, 2003; Oh, Draper, \& Dixon, 2009). Una muestra de 133 visitantes genera un margen de error del 7\% con un nivel de confianza del 90\%, según la calculadora de muestras de la Facultad de Medicina de la UNNE (Argentina) (http://www.med.unne.edu. ar/biblioteca/calculos/calculadora.htm).

La valoración de la nitidez del agua obtuvo una puntuación media de 3,04 puntos sobre 5. Un 54\% de los encuestados contestaron que la nitidez del agua no era un factor determinante en la valoración de los paisajes analizad os. Del 46\% restante, sólo un 19\% mantuvieron la respuesta afirmativa después de la explicación sobre la calidad ecológica del agua y su turbidez. Así pues, un $81 \%$ prefirieron la calidad ecológica a la nitidez, por lo que la nitidez del agua no se incorporó como variable en la calidad escénica.

Con el objetivo de calcular el índice de calidad escénica se utilizaron las ponderaciones para cada sentido obtenidas durante el proceso de elaboración de las encuestas. El sentido de la vista obtuvo una puntuación media de 4,77, el de oído de 3,88 y el de olfato de 3,42. En lo que se refiere a las puntuaciones de cada espacio, "les Basses del Ter Vell" fue el paisaje que obtuvo mejor puntuación, seguido por "les Basses d'en Coll". "La Pletera" fue el paisaje que obtuvo una puntuación inferior. A pesar de todo, las diferencias entre los tres paisajes no resultaron demasiado acentuadas (tabla 3). La pregunta sobre valoración general del paisaje realizada durante la encuesta permitió validar los resultados del índice de calidad escénica. El test de correlación presentó un valor de $\mathrm{R}=0,97$ con un p-valor de 0,14.

Tabla 3. Puntuaciones obtenidas para cada paisaje

\begin{tabular}{lrrr}
\hline & Basses del Ter Vell & La Pletera & Basses d'en Coll \\
\hline Índice de calidad escénica & & & \\
\hline Puntuación paisaje visual & 0,57 & 0,51 & 0,62 \\
Puntuación paisaje sonoro & 0,61 & 0,57 & 0,52 \\
Puntuación paisaje olfativo & 0,78 & 0,82 & 0,78 \\
Índice de calidad escénica & 0,95 & 0,90 & 0,94 \\
Valoración encuesta & 4,49 & 4,02 & 4,50 \\
\hline Potencial como recurso turístico & & & \\
\hline Presencia en medios de difusión turística & 1 & 1 & 1 \\
Valor cultural & 1 & 1 & 2 \\
Distancia desde núcleos turísticos & 3 & 2 & 3 \\
Señalización de acceso & 1 & 2 & 1 \\
Cantidad de caminos & 1 & 2 & 2 \\
Paneles explicativos & 2 & 2 & 3 \\
Equipamiento turístico & 2 & 1 & 2 \\
Figura de protección & 3 & 3 & \\
Potencial turístico & 1,75 & 1,75 & \\
\hline
\end{tabular}

Elaboración propia.

El paisaje que presenta un mayor potencial como recurso turístico es "les Basses d'en Coll" con una puntación de 2 sobre 3 (tabla 3). Los otros dos espacios se situaron un poco por debajo, con una puntuación de 1,75 cada uno. "Les Basses d'en Coll destaca en cuanto a calidad de la señalización. "Les Basses del Ter Vell presentó su mejor puntuación en la distancia desde núcleos turísticos, mientras que la Pletera no destaca especialmente en ningún aspecto. Los tres espacios obtuvieron la puntuación máxima en el ítem de figura de protección debido a que los tres son reservas naturales parciales.

Con los dos resultados obtenidos para cada espacio, se pudo elaborar el diagrama de tipificación de los paisajes del agua (figura 4). Para remarcar las diferencias entre cada espacio, se utilizó una escala relativa en lugar de los valores absolutos (entre 0 y 3 ), por lo que los umbrales vienen determinados por la mediana de los tres valores. Así pues, cada espacio quedó localizado en un cuadrante diferente. "Les Basses d'en Coll" se situó en el cuadrante de alto potencial turístico y alta calidad escénica, "les Basses del Ter Vell" en el cuadrante de alta calidad escénica y bajo potencial turístico y "la Pletera" en el cuadrante con bajo potencial turístico y baja calidad escénica. 


\section{Discusión}

Los resultados en relación a la preferencia de calidad ecológica del agua versus su calidad visual presentan cierta divergencia con determinados estudios previos, donde la nitidez del agua era un factor importante en la valoración del paisaje (Keeler et al., 2015; Poor et al., 2001; Wilson \& Carpenter, 1999) se puede explicar por el hecho que en estos estudios el agua tiene una función recreativa importante (nadar, paseos en barca, etc.), a diferencia de los humedales analizados en nuestro caso, donde el agua no puede ser utilizada para fines recreativos, ya sea por la poca profundidad o por las restricciones de uso. También es influyente la proximidad a la playa, que ofrece una zona recreativa acuática con muchas más posibilidades.

Figura 1. Resultados del diagrama de tipificación de los paisajes

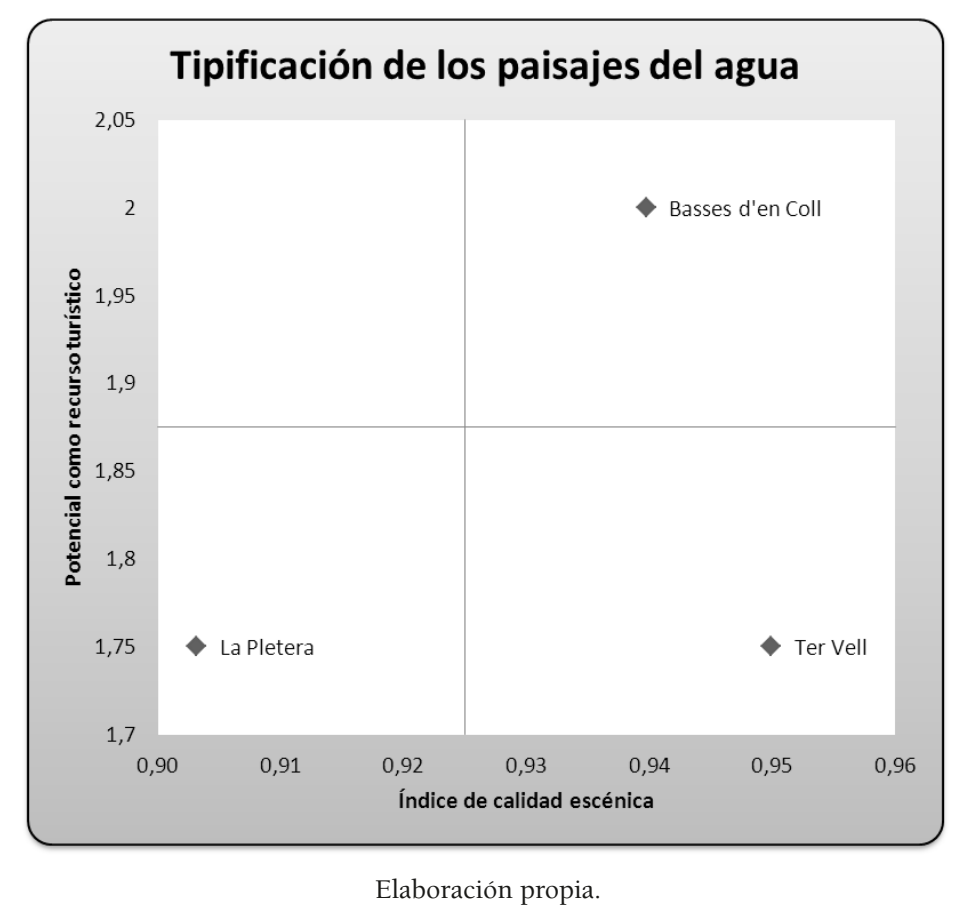

En línea con la primacía que tiene la componente visual de los paisajes, tanto en el mundo científico como en el imaginario colectivo, fue también el sentido que obtuvo una mayor puntuación en las encuestas, obteniendo una puntación de 4.77 sobre 5 . No obstante, y contrario a lo que cabría esperar por el trato recibido por la comunidad científica, los sentidos del oído y del olfato también aparecen como importantes para los visitantes de los humedales, coincidiendo con algunos estudios que ya enfatizaban la importancia de estos sentidos cuando se valora un paisaje (Henshaw, 2013; Schafer, 1977). Así pues, analizando cada uno de los componentes del paisaje: visual, sonoro y olfativo; se observa que cada paisaje dominó en uno de los sentidos. "Les Basses del Ter Vell" es el que presentó el paisaje sonoro de mejor calidad, seguramente por el hecho que es el menos visitado y, por ello, más tranquilo. "La Pletera" fue el paisaje mejor valorado en la dimensión olfativa, las respuestas relacionadas con el olor a mar fueron más abundantes en este espacio, un olor que es muy valorada por los visitantes. Mientras que en, por ejemplo, "les Basses d'en Coll" solían notarse a menudo olores relacionados con la actividad agraria o con aguas encharcadas, que también podían olerse en "Les Basses del Ter Vell". "Les Basses d'en Coll" obtuvo la mejor puntuación en la dimensión visual, probablemente porque es el paisaje con una mayor vocación agraria y con una mayor presencia de agua, paisajes que, por lo general, son mejor valorados por los turistas (Real et al., 2000). La vista también fue el sentido que obtuvo una mayor puntuación, constatándose que en la percepción del paisaje existe una dominancia del sentido de la vista, reforzado por el enfoque estético de las culturas occidentales, también dominado por la vista (Prior, 2016). Sin embargo, esto difiere con algunos estudios previos que muestran que una de las cualidades más valoradas por los visitantes de estos paisajes del agua es la tranquilidad (Pueyo-Ros, Ribas, \& Fraguell, 2016b) un aspecto que está claramente ligado al sentido del oído (Filipan et al., 2017). 
Aunque una muestra de tres espacios resulta insuficiente para sacar conclusiones definitivas, el índice de correlación utilizado para comprobar la validez de la metodología sugiere que el método puede generar buenos resultados, aunque será necesario aplicarlo en más espacios para comprobar si el índice de calidad escénica continúa correlacionándose con éxito con las valoraciones de los visitantes.

En relación al potencial como recurso turístico, aun habiendo obtenido la misma puntuación, los dos espacios difieren en muchos de los ítems valorados. Cada uno de los ítems por separado se puede utilizar como guía para realizar actuaciones en los espacios a fin de mejorar su potencial turístico. Por ejemplo, los resultados muestran que en "les Basses del Ter Vell" hay un problema de señalización para acceder al lugar. En "la Pletera", por otro lado, sería más prioritario invertir en equipamiento turístico, por ejemplo. Es remarcable que los tres espacios carecen de relevancia en las guías turísticas, demostrando el poco interés de los promotores turísticos de la región en potenciar este tipo de atracciones turísticas.

El diagrama de tipificación resultante es coherente con lo que cabría esperar atendiendo a la tipología y estado de conservación de cada uno de ellos. Especialmente en el caso de "la Pletera", un espacio altamente degradado debido a un incipiente proceso de urbanización interrumpido desde la década de 1990. Aunque "les Basses d'en Coll" sea el espacio mejor valorado, la frecuencia de visitantes observada es mayor en los otros dos espacios, esto es debido a que "les Basses d'en Coll" es el único espacio que no tiene acceso a la playa. Mientras que "la Pletera", aun siendo un espacio degradado tiene una gran afluencia de visitantes debido a que da acceso a una playa poco masificada con facilidad de aparcamiento, aunque sólo el 12\% de visitantes visita el espacio por sus valores naturales (Pueyo-Ros, Ribas, \& Fraguell, 2016a).

El hecho de que "les Basses d'en Coll" se localice en el cuadrante superior derecho pero no tenga una gran afluencia de visitantes demuestra que las tareas de promoción no se están efectuando correctamente. El espacio está capacitado para convertirse en un importante recurso turístico, pero la administración está perdiendo una oportunidad para reducir la estacionalidad y la saturación en las playas ofreciendo un complemento al turismo de sol y playa. "Les Basses del Ter Vell", por otro lado, presentan una buena calidad escénica pero no disponen de los atributos necesarios para convertirse en un reclamo turístico. Sus puntos débiles son principalmente la señalización de acceso y la falta de caminos para recorrer el espacio, pues este sólo se puede visitar desde la periferia o desde un único mirador. "La Pletera" es un caso aparte, aunque los resultados de la tipificación recomendarían no invertir recursos en el espacio desde la perspectiva turística, el alto valor ecológico del espacio debido a ser el hábitat de especies endémicas amenazadas como el fartet (Aphanius iberus) le ha permitido beneficiarse de un proyecto Life que restaurará ambientalmente el espacio, creará lagunas y retirará los restos del proceso de urbanización. Este proyecto puede aumentar la calidad escénica del espacio, pero a la vez reducir el número de visitantes actuales pues los accesos a la playa estarán más limitados al consolidar el papel de este espacio cómo reserva natural parcial (Pueyo-Ros et al., 2016a).

Por último, es importante destacar que en este estudio sólo se valora la función social de los paisajes, olvidando las funciones ecológicas de los ecosistemas que forman el paisaje, que a menudo pueden ser conflictivas con la apreciación estética (Gobster, 1999; Prior, 2016). El aprovechamiento de estos espacios como recurso turístico debe tomar en consideración las funciones ecológicas, como proveer de hábitat o ser punto de nidificación de muchas especies. No podemos olvidar que el objetivo principal de los espacios naturales protegidos es la conservación del patrimonio natural. Por esta razón, los usos turísticos y recreativos no pueden menoscabar o perjudicar este principio fundamental. La gestión de estos espacios naturales debe procurar la compatibilidad y la sostenibilidad de los distintos usos de una forma concertada con los agentes sociales implicados en la gestión del territorio.

\section{Conclusiones}

En este trabajo se ha presentado un método para evaluar los paisajes del agua desde una doble perspectiva estética y turística, aplicándolo en tres casos de estudio. Se ha valorado su calidad escénica y su potencial como recurso turístico a la vez que se relacionan los resultados para obtener una tipificación que permita sistematizar las necesidades de cada espacio y la mejor forma de gestionarlos desde el punto de vista turístico. Los resultados se muestran coherentes con las características de cada uno de los espacios. De todas formas, la aplicación a sólo tres espacios es insuficiente para sacar conclusiones definitivas. Sin embargo, tanto los resultados generales como la validación de los mismos dan el crédito necesario para seguir aplicando la metodología a nuevos paisajes del agua del litoral mediterráneo. 
Los resultados que se pueden obtener con la metodología presentada sirven de guía para la gestión turística de espacios naturales. Además de generar un catálogo de recursos tipificados en función de su calidad y capacidad, también permiten disponer de una pauta donde ver que intervenciones son más necesarias o prioritarias. Los resultados muestran en qué espacios es más eficiente intervenir y con qué medidas: difusión, mejora de equipamientos, etc.

Hasta donde llega nuestro conocimiento, no hay estudios previos que desarrollen una metodología parecida a la presentada en este trabajo. Existen gran cantidad de estudios dedicados a evaluar la calidad escénica del paisaje considerando sólo la dimensión visual (e.g. Arriaza et al., 2004; Ezquerra \& Navarra, 2015; Loures, Loures, Nunes, \& Panagopoulos, 2015). Otros estudios también se han dedicado a analizar la calidad sonora de los paisajes (e.g. Iglesias Merchan et al., 2014; Rey Gozalo, Trujillo Carmona, Barrigón Morillas, Vílchez-Gómez, \& Gómez Escobar, 2015) pero ningún estudio ha intentado hasta ahora evaluar un paisaje considerando los sentidos de la vista, el oído y el olfato al mismo tiempo. Por otro lado, mientras existen multitud de estudios dedicados a evaluar el potencial turístico de determinados paisajes (e.g. Cebrián \& García, 2010; Prat \& Cànoves, 2012), no se ha podido encontrar ningún estudio que relacione los dos conceptos para realizar una tipificación de los recursos tal y como se ha presentado en este artículo.

Sin embargo, el método presentado representa sólo un punto de partida para empezar a concebir los paisajes del agua de forma integral, dejando atrás la concepción del paisaje como fotografía y entenderlo como un espacio que tanto los visitantes como la población local viven y perciben con todos los sentidos. Si queremos que los paisajes del agua se conviertan en recursos turísticos de calidad en los destinos litorales, es necesario que presenten las condiciones óptimas para garantizar una buena experiencia turística y recreativa a los visitantes, preservando a la vez los valores intrínsecos que permitan asegurar su perdurabilidad.

\section{Agradecimientos}

Los autores quieren expresar su agradecimiento a Maria Blanco por su colaboración en la elaboración de las encuestas. También a todos los participantes que colaboraron de buena voluntad contestando las encuestas. Este trabajo es parte del proyecto Análisis de tendencias y estrategias de resiliencia en los usos y el consumo de agua en espacios turísticos. El caso de la Costa Brava [CSO2013-41262-P] financiado por el Ministerio de Ciencia y Tecnología del Gobierno de España. El Ministerio de Educación y Cultura del Gobierno de España financia la beca predoctoral del primer autor [FPU13/00663].

\section{Referencias}

Alcaraz, C., Pou-Rovira, Q., \& García-Berthou, E. (2007). Use of a flooded salt marsh habitat by an endangered cyprinodontid fish (Aphanius iberus). Hydrobiologia, 600(1), 177-185. https://doi. org/10.1007/s10750-007-9230-y

Arriaza, M., Cañas-Ortega, J. F., Cañas-Madueño, J. a., \& Ruiz-Aviles, P. (2004). Assessing the visual quality of rural landscapes. Landscape and Urban Planning, 69(October 2003), 115-125. https://doi. org/10.1016/j.landurbplan.2003.10.029

Borer, M. I. (2013). Being in the City: The Sociology of Urban Experiences. Sociology Compass, 7(11), 965-983. https://doi.org/10.1111/soc4.12085

Brown, T. C., \& Daniel, T. C. (1991). Landscape aesthetics of riparian environments: relationship of flow quantity to scenic quality along a wild and scenic river. Water Resources Research, 27(8), 1787-1795. https://doi.org/10.1029/91WR00975

Burger, J. (2003). Assessing perceptions about ecosystem health and restoration options in three east coast estuaries. Environmental Monitoring and Assessment, 83(2), 145-162. https://doi. org/10.1023/A:1022505300319

Burmil, S., Daniel, T. C., \& Hetherington, J. D. (1999). Human values and perceptions of water in arid landscapes. Landscape and Urban Planning, 44(2-3), 99-109. https://doi.org/10.1016/S01692046(99)00007-9

Büscher, B., \& Fletcher, R. (2016). Destructive creation: capital accumulation and the structural violence of tourism. Journal of Sustainable Tourism, 1-17. https://doi.org/10.1080/09669582.2016.1159214 
Butler, R. W. (1980). The concept of a tourist area cycle of evolution: implications for management of resources. Canadian Geographer. https://doi.org/10.1111/j.1541-0064.1980.tb00970.x

Butler, R. W. (Ed.). (2006). The tourism area life cycle. Channel View Publications.

Butler, R. W. (2011). Tourism area life cycle. Contemporary Tourism Reviews.

Cebrián, F., \& García, J. A. (2010). Propuesta metodológica para la identificación, clasificación y puesta en valor de los recursos territoriales del turismo interior. La provincia de Albacete. Boletín de la Asociación de Geógrafos Españoles, 54, 361-383.

Chan, K. M. A., Satterfield, T., \& Goldstein, J. (2012). Rethinking ecosystem services to better address and navigate cultural values. Ecological Economics, 74, 8-18. https://doi.org/10.1016/j. ecolecon.2011.11.011

Daniel, T. C. (2001). Whither scenic beauty? Visual landscape quality assessment in the 21st century. Landscape and Urban Planning, 54, 267-281. https://doi.org/10.1016/S0169-2046(01)00141-4

Daniel, T. C., \& Boster, R. S. (1976). Measuring landscape esthetics: the scenic beauty estimation method. USDA Forest Service Research Paper.

Dann, G., \& Jacobsen, J. K. S. (2003). Tourism smellscapes. Tourism Geographies, 5(1), 3-25. https://doi. org/10.1080/1461668032000034033

de la Fuente de Val, G., Atauri, J. a., \& de Lucio, J. V. (2006). Relationship between landscape visual attributes and spatial pattern indices: A test study in Mediterranean-climate landscapes. Landscape and Urban Planning, 77(4), 393-407. https://doi.org/10.1016/j.landurbplan.2005.05.003

Dhakal, S. P. (2014). An exploratory perspective on sustainable tourism as wise-use of a protected wetland in Thailand. International Journal of Tourism Policy, 5(3), 159. https://doi.org/10.1504/ IJTP.2014.063079

Dramstad, W. E., Tveit, M. S., Fjellstad, W. J., \& Fry, G. L. a. (2006). Relationships between visual landscape preferences and map-based indicators of landscape structure. Landscape and Urban Planning, 78(4), 465-474. https://doi.org/10.1016/j.landurbplan.2005.12.006

Ezquerra, A., \& Navarra, M. (2015). Metodología de valoración de paisaje empleando ArcGIS : Aplicación a las Vías Pecuarias. Informes de la Construcción, 67(537). https://doi.org/10.3989/ic.13.051

Farina, A. (2014). Soundscape ecology: principles, patterns, methods and applications. Dordrecht [etc.]: Springer. https://doi.org/10.1007/978-94-007-7374-5

Ferrario, F. F. (1979). The Evaluation of Tourist Resources: an Applied Methodology. Journal of Travel Research. https://doi.org/10.1177/004728757901700405

Filipan, K., Boes, M., De Coensel, B., Lavandier, C., Delaitre, P., Domitrović, H., \& Botteldooren, D. (2017). The Personal Viewpoint on the Meaning of Tranquility Affects the Appraisal of the Urban Park Soundscape. Applied Sciences, 7(1), 91. https://doi.org/10.3390/app7010091

Fletcher, R. (2009). Ecotourism discourse: challenging the stakeholders theory. Journal of Ecotourism, 8(3), 269-285. https://doi.org/10.1080/14724040902767245

Germino, M. J., Reiners, W. a., Blasko, B. J., McLeod, D., \& Bastian, C. T. (2001). Estimating visual properties of Rocky Mountain landscapes using GIS. Landscape and Urban Planning, 53(1-4), 71-83. https://doi.org/10.1016/S0169-2046(00)00141-9

Gimblett, H. R., Itami, R. M., \& Fitzgibbon, J. E. (1985). Mystery in an Information Processing Model of Landscape Preference. Landscape Jrnl., 4(2), 87-95. https://doi.org/10.3368/lj.4.2.87

Gobster, P. H. (1999). An ecological aesthetic for forest landscape management. Landscape Journal, 18(1), 54-64. https://doi.org/10.1086/250095

Gobster, P. H., Nassauer, J. I., Daniel, T. C., \& Fry, G. (2007). The shared landscape: What does aesthetics have to do with ecology? Landscape Ecology, 22(7), 959-972. https://doi.org/10.1007/s10980-0079110-x

Henshaw, V. (2013). Urban Smellscapes. Urban Smellscapes: Understanding and Designing City Smell Environments. Routledge. https://doi.org/10.4324/9780203072776

Herzog, T. R. (1985). A cognitive analysis of preference for waterscapes. Journal of Environmental Psychology, 5, 225-241. https://doi.org/10.1016/S0272-4944(85)80024-4 
Hetherington, J., Daniel, T. C., Brownt, T. C., Forest, R. M., \& Station, R. E. (1993). Is Motion More Important Than It Sounds ?: the Medium of Presentation in Environment Perception Research. Journal of Environmental Psychology, 13, 283-291. https://doi.org/10.1016/S0272-4944(05)80251-8

Ibàñez, J. J., \& Burriel, J. Á. (2010). Mapa de cubiertas del suelo de cataluña: características de la tercera edición y relación con siose. Tecnologías de la Información Geográfica: La Información Geográfica al servicio de los ciudadanos, 3, 179-198.

Iglesias Merchan, C., Diaz-Balteiro, L., \& Soliño, M. (2014). Noise pollution in national parks: Soundscape and economic valuation. Landscape and Urban Planning, 123, 1-9. https://doi.org/10.1016/j. landurbplan.2013.11.006

Institut Cartogràfic i Geològic de Catalunya [ICGC] (2016). LEstartit [Mapa] 1:50.000. En Mapa topogràfic 1:50.000.

Kaltenborn, B. P., \& Bjerke, T. (2002). Associations between environmental value orientations and landscape preferences. Landscape and Urban Planning, 59(1), 1-11. https://doi.org/10.1016/S01692046(01)00243-2

Kaplan, R., \& Kaplan, S. (1989). The Experience of nature : a psychological perspective. Cambridge [etc.] : Cambridge University Press.

Keeler, B. L., Wood, S. a, Polasky, S., Kling, C., Filstrup, C. T., \& Downing, J. a. (2015). Recreational demand for clean water: evidence from geotagged photographs by visitors to lakes. Frontiers in Ecology and the Environment, 150129065716004. https://doi.org/10.1890/140124

Kim, S. O., \& Shelby, B. (2011). Effects of information on perceived crowding and encounter norms. Environmental Management, 47, 876-884. https://doi.org/10.1007/s00267-011-9654-z

Kušen, E. (2010). A system of tourism attractions. Tourism, 58(4), 409-425.

Lew, A. a. (1987). A framework of tourist attraction research. Annals of Tourism Research, 14(4), 553-575. https://doi.org/10.1016/0160-7383(87)90071-5

Loures, L., Loures, A., Nunes, J., \& Panagopoulos, T. (2015). Landscape Valuation of Environmental Amenities throughout the Application of Direct and Indirect Methods. Sustainability, 7(1), 794-810. https://doi.org/10.3390/su7010794

Łowicki, D., \& Piotrowska, S. (2015). Monetary valuation of road noise . Residential property prices as an indicator of the acoustic climate quality. Ecological Indicators, 52, 472-479. https://doi.org/10.1016/j. ecolind.2015.01.002

Mata, R., \& Fernández, S. (2010). Paisajes y patrimonios culturales del agua. La salvaguarda del valor patrimonial de los regadíos tradicionales. Scripta Nova: Revista electrónica de geografía y ciencias sociales, 14(14), 323-339.

Mercado-Alonso, I., Fernández-Tabales, A., \& Muñoz-Yules, O. (2017). Perceptions and social valuations of landscape. Objectives and methodology for citizen participation in landscape policies. Landscape Research, 1-17. https://doi.org/10.1080/01426397.2017.1302570

Miller, D. (2001). A method for estimating changes in the visibility of land cover. Landscape and Urban Planning, 54(September 2000), 91-104. https://doi.org/10.1016/S0169-2046(01)00128-1

Ministerio de Cultura, Empresa y Turismo [MCET] (2008). Manual para la formulación del inventario de recursos turísticos a nivel nacional. San Isidro.

Muñoz, M. D., Pérez, L., Sanhueza, R., Rovira, A., \& Urrutia, R. (2006). Los paisajes del agua en la cuenca del río Baker : bases conceptuales para su valoracion integral. Revista de Geografía Norte Grande, 36, 31-48.

Navrátil, J., \& Pícha, K. (2013). Factors influencing the imposition of a charge on the entrance to the interpretive trails in the large protected areas. Acta Universitatis Agriculturae et Silviculturae Mendelianae Brunensis, 61(4), 1041-1049. https://doi.org/10.11118/actaun201361041041

Nogué, J., Puigbert, L., Sala, P., \& Bretcha, G. (2010). Paisatge i participació ciutadana : l'experiència dels catàlegs de paisatge de Catalunya. Olot : Observatori del Paisatge de Catalunya.

Oh, C.-O., Draper, J., \& Dixon, A. W. (2009). Assessing Tourists' Multi-Attribute Preferences for Public Beach Access. Coastal Management, 37(2), 119-135. https://doi.org/10.1080/08920750802701128 
Poor, P., Boyle, K. J., Taylor, L., \& Bouchard, R. (2001). Objective versus subjective measures of water clarity in hedonic property value models. Land Economics. https://doi.org/10.2307/3146935

Prat, J. M., \& Cànoves, G. (2012). El turismo cultural como oferta complementaria en los destinos de litoral . El caso de la Costa Brava (España). Investigaciones Geográficas, (79), 119-135.

Prior, J. (2016). Sonic environmental aesthetics and landscape research. Landscape Research. https://doi.or g/10.1080/01426397.2016.1243235

Pueyo-Ros, J., Ribas, A., \& Fraguell, R. M. (2016a). Impacts of restoration projects in seaside wetlands on tourism sector of Costa Brava (Spain). En S. Lira, A. Mano, C. Pinheiro, \& R. Amoêda (Eds.), Tourism 2016. International Conference on Global Tourism and Sustainability (pp. 315-323). Lagos: Greenlines Institute.

Pueyo-Ros, J., Ribas, A., \& Fraguell, R. M. (2016b). Recreational uses and motivations of visitors in seaside wetlands of Costa Brava (Spain). International Journal of Sustainable Development and Planning, 11(3), 385-395. https://doi.org/10.2495/SDP-V11-N3-385-395

Real, E., Arce, C., \& Manuel Sabucedo, J. (2000). Classification of Landscapes Using Quantitative and Categorical Data, and Prediction of Their Scenic Beauty in North-Western Spain. Journal of Environmental Psychology, 20, 355-373. https://doi.org/10.1006/jevp.2000.0184

Rey Gozalo, G., Trujillo Carmona, J., Barrigón Morillas, J. M., Vílchez-Gómez, R., \& Gómez Escobar, V. (2015). Relationship between objective acoustic indices and subjective assessments for the quality of soundscapes. Applied Acoustics, 97, 1-10. https://doi.org/10.1016/j.apacoust.2015.03.020

Sardà, R., Mora, J., Ariza, E., Avila, C., \& Jimenez, J. A. (2009). Decadal shifts in beach user sand availability on the Costa Brava (Northwestern Mediterranean Coast). Tourism Management, 30(2), 158-168. https://doi.org/10.1016/j.tourman.2008.05.011

Schafer, R. M. (1977). The Tuning of the world. New York: Alfred A. Knopf.

Schirpke, U., Tasser, E., \& Tappeiner, U. (2013). Predicting scenic beauty of mountain regions. Landscape and Urban Planning, 111, 1-12. https://doi.org/10.1016/j.landurbplan.2012.11.010

Sliwa, M., \& Riach, K. (2011). Making Scents of Transition: Smellscapes and the Everyday in «Old» and «New» Urban Poland. Urban Studies, 49(1), 23-41. https://doi.org/10.1177/0042098011399596

Söderqvist, T., Mitsch, W. J., \& Turner, R. K. (2000). Valuation of wetlands in a landscape and institutional perspective. Ecological Economics, 35, 1-6. https://doi.org/10.1016/S0921-8009(00)00163-4

Stamps, A. E. (2004). Mystery, complexity, legibility and coherence: A meta-analysis. Journal of Environmental Psychology, 24(1), 1-16. https://doi.org/10.1016/S0272-4944(03)00023-9

Urry, J. (2002). The Tourist Gaze. London: Sage Publications.

Van den Broeck, M., Waterkeyn, A., Rhazi, L., Grillas, P., \& Brendonck, L. (2015). Assessing the ecological integrity of endorheic wetlands, with focus on Mediterranean temporary ponds. Ecological Indicators, 54, 1-11. https://doi.org/10.1016/j.ecolind.2015.02.016

Vila, J., Rodríguez-Carreras, R., Varga, D., Ribas, A., Úbeda, X., Asperó, F., ... Outeiro, L. (2015). Stakeholder Perceptions of Landscape Changes in the Mediterranean Mountains of the North-Eastern Iberian Peninsula. Land Degradation \& Development, n/a-n/a. https://doi.org/10.1002/ldr.2337

Wilson, M., \& Carpenter, S. (1999). Economic valuation of freshwater ecosystem services in the United States: 1971-1997. Ecological applications, 9(3), 772-783. https://doi.org/10.2307/2641328 